\title{
ANÁLISIS DE LAS NOCHES TROPICALES EN LA FACHADA ATLÁNTICA DE LA PENÍNSULA IBÉRICA. UNA PROPUESTA METODOLÓGICA
}

\author{
Dominic Royé \\ Alberto Martí Ezpeleta \\ Departamento de Geografía. Universidad de Santiago de Compostela \\ dominic.roye@usc.es ; alberto.marti@usc.es
}

\section{RESÚMEN}

En este trabajo se aplica una metodología nueva al estudio de las noches calurosas, también denominadas «tropicales», en Galicia y en Portugal de cara a identificar aquellas noches en las que la población pueda verse afectada por estrés térmico. La utilización de dos indicadores obtenidos a través de datos semihorarios ha permitido definir con más detalle las características térmicas de las noches entre mayo y octubre, pudiendo así evaluar con más precisión el riesgo para el bienestar y la salud de la población. Se produce un importante aumento de la frecuencia de noches tropicales y noches cálidas en la fachada atlántica, desde el norte de Galicia hasta el sur de Portugal. La menor latitud y la proximidad al litoral están relacionadas con la mayor persistencia del calor y del estrés térmico durante estas noches. En áreas de interior la persistencia es menor. Las noches calurosas son más frecuentes e intensas en el centro de las ciudades, por el efecto de la isla de calor urbana.

Palabras clave: Noche tropical, estrés térmico, isla de calor, Galicia, Portugal.

\begin{abstract}
This paper presents a new methodology for the study of warm nights, also called «tropical», in Galicia and Portugal in order to identify those nights where people can be affected by heat stress. The use of two indicators obtained through half-hourly data has allowed us to define in more detail the thermal characteristics of the nights between May and October, the-
\end{abstract}

Fecha de recepción: noviembre 2013.

Fecha de aceptación: diciembre 2014. 
reby being able to more accurately assess the risk to the health and well-being of the population. There is a significant increase in the frequency of tropical nights and warm nights on the Atlantic coast, from the north of Galicia to the south of Portugal. The lower latitude and proximity to the coastline are associated with greater persistence of heat and thermal stress during these nights. In inland areas the persistence is less. The warmest nights are more frequent and intense in centres of the cities, due to the effect of the urban heat island.

Keywords: Tropical night, heat stress, heat island, Galicia, Portugal.

\section{INTRODUCCIÓN}

Durante el periodo estival las olas de calor constituyen un fenómeno recurrente en los climas templados de Europa, especialmente en las regiones más meridionales y más expuestas a las masas de aire cálidas procedentes del norte de África, causantes de dichos episodios. Se trata de un fenómeno temporal y espacial muy variable, que repercute negativamente tanto en el bienestar como en la salud de la población. Los efectos de las olas de calor en la población han sido descritos por diferentes autores, que han encontrado relaciones evidentes entre las altas temperaturas y la mortalidad (Huynem et al. 2001, Díaz et al. 2002, 2006, GarcíaHerrera et al. 2005, WHO 2004). En la última década se ha podido observar a escala global un considerable aumento de estos episodios (Coumou y Robinson 2013, Coumou y Rahmstorf 2012). Sin duda, la ola de calor más significativa desde hace 500 años afectó a Europa en el año 2003 causando una elevada mortalidad (Coumou y Rahmstorf 2012). En Francia, por ejemplo, se alcanzaron los 15.000 muertos debidos a las altas temperaturas entre el uno y el veinte de agosto de 2003 (Fouillet et al.2006). En España las cifras oficiales hablaron de 141, pero el Centro Nacional de Epidemiología cifra en 6.500 el número de fallecimientos atribuibles al calor. En la mayoría de las ciudades del interior y del Mediterráneo se alcanzaron máximas por encima de los $40^{\circ} \mathrm{C}$ y mínimas muy superiores a los $20^{\circ} \mathrm{C}$. En el último informe del Grupo de Trabajo II del IPCC (2014) señala que hay evidencia de mayor mortalidad relacionada con el calor y menor con el frío en algunas zonas del planeta como Europa, y se advierte del muy probable incremento de la frecuencia e intensidad de los episodios de calor extremo que conllevará el calentamiento global.

El IPCC destaca también como muy probable que los días y noches cálidos sean más numerosos y más cálidos, y no siempre asociados a las olas de calor. Durante estos días, los efectos percibidos por la población son los provocados por los extremos de temperatura, así como por la diferencia que se produce entre la máxima y la mínima. Diversos estudios muestran relaciones significativas entre un mayor riesgo de mortalidad y una menor amplitud térmica debida a elevados valores de las temperaturas mínimas (Rooney et al. 1995, Gritze et al.2005), pues en dichos casos el estrés térmico persiste y se agrava debido a que se impide al organismo un descanso nocturno de las altas temperaturas diurnas.

La consecuencia más habitual de las noches calurosas sobre la salud es su impacto en el sueño y en el descanso de las personas. El calor puede llevar, entre otros efectos, a alteraciones y privaciones de éste debidos a la puesta en marcha de los procesos de termorregulación (Buguet 2007). Concretamente, las temperaturas superiores a las del confort 
pueden influir en el aumento del desvelo y en la disminución de las fases REM (Rapid Eye Movement) y SWS (Slow-wave Sleep) (Haskella et al. 1981, Okamoto y Mizuno 2005).

Las alteraciones del sueño se dan con mayor incidencia, al igual que el riesgo general de mortalidad en olas de calor, en personas con avanzada edad (Buguet 2007, Koppe et al. 2004). Sin embargo, hay que tener en cuenta que no sólo la temperatura es la causante de estos impactos negativos, sino que también influyen la humedad y otros elementos atmosféricos, así como otras variables ambientales, como el nivel de ruido o el campo electromagnético (Muzet 2007). Claro que en estos tipos de análisis tampoco se debe excluir otros determinantes de tipo social e individual, pues el estrés térmico depende, por un lado, del tipo de cama o/y de la ropa, y por otro, entre otras cosas, de la construcción: la ventilación, la orientación de la vivienda, tipos de materiales y la conducción de calor entre interior y exterior (Höppe y Martinac 1998). Y en los casos de presencia de aparatos de aire acondicionado, éstos hacen que en la correlación entre factores ambientales y calidad del sueño prevalezcan las causas socioeconómicas, como ha demostrado O'Neill et al. (2005) para varias ciudades estadounidenses.

Precisamente en las ciudades los impactos negativos del calor sobre el confort y la salud se ven agravados por el fenómeno urbano de «isla de calor». Temperaturas más elevadas que en el entorno, favorecidas por diversos factores urbanos (Moreno 1999, López et al. 1993), y el más lento enfriamiento nocturno, generan durante los episodios de calor unas condiciones de mayor estrés térmico y el aumento del riesgo para la salud de los habitantes de las ciudades, en contraste con el medio rural circundante.

Teniendo en cuenta las proyecciones de cambio climático, en las que se estima una mayor frecuencia, duración e intensidad de las olas de calor que afectarán a Europa, con mayores impactos en la Península Ibérica y en las regiones del Mediterráneo (Fischer y Schär 2010, IPCC 2014), consideramos de interés poder disponer de indicadores adecuados que permitan evaluar el riesgo de impacto del calor nocturno sobre la salud y el bienestar de la población. Es por ello que el objetivo de este trabajo consiste en aplicar una metodología nueva al estudio de las noches calurosas, también denominadas «tropicales», en Galicia y en Portugal de cara a identificar aquellas noches en las que la población pueda verse afectada por estrés térmico.

\section{EL CONCEPTO DE NOCHE TROPICAL}

El concepto de «noche tropical» ha sido definido como una noche en la que la temperatura mínima es superior o igual a los $20^{\circ} \mathrm{C}$ (Vincent et al. 2005, Alexander et al. 2006, WMO 2009, EEA Report 2012, Donat et al. 2013, DWD 2013). El Expert Team on Climate Change Detection and Indices utiliza los días con temperaturas mínimas $>20^{\circ} \mathrm{C}$ como uno de los indicadores climáticos para el seguimiento del cambio climático (Lisa et al. 2009, Russo y Sterl 2011). Se trata de un índice de umbral, que pertenece a un grupo de índices en el que también se integran otros como días de fuerte precipitación o días de helada. En estos índices se fijan unos umbrales para identificar los días en los que se superan o no se alcanzan éstos. Como consecuencia, estos umbrales fijos no pueden ser válidos para todas las zonas climáticas del planeta (Alexander et al. 2006). En el caso de las noches tropicales es evidente que su umbral de $20^{\circ} \mathrm{C}$ puede ser útil en Europa, pero para un clima tropical con temperaturas 
mínimas superiores a éstos durante varios meses, sería cuestionable. Un ejemplo ilustrativo al respecto de este problema puede verse en la página web del servicio meteorológico de Hong Kong (Hong Kong Observatory 2012). En ella no se habla de «noches tropicales» sino de «hot nights»; y, en segundo lugar, el umbral aplicado se define como igual o superior a $28^{\circ} \mathrm{C}$. Incluso en un estudio sobre los efectos biometeorológicos de las temperaturas nocturnas de Atenas (Nastos y Matzarakis 2008), se usó una temperatura mínima de $23^{\circ} \mathrm{C}$ como umbral. La mención de «tropical» es, pues, una referencia a una situación no habitual para las regiones extratropicales, como es el caso de Europa.

Hemos observado que el uso exclusivo de la temperatura mínima como referencia para delimitar las noches tropicales, puede resultar insuficiente para llevar a cabo un análisis detallado del impacto del calor nocturno en la salud. Por una parte hay que tener en cuenta que la temperatura mínima suele alcanzarse habitualmente en los momentos próximos a la salida del sol; pero se pueden registrar temperaturas superiores a $20^{\circ} \mathrm{C}$ durante muchas horas de la noche y obtener, como registro mínimo, una temperatura inferior a los $20^{\circ} \mathrm{C}$. En estos casos el estrés térmico no es necesariamente menor que en los días con una temperatura mínima igual o superior a $20^{\circ} \mathrm{C}$, sobre todo si tenemos en cuenta que la fase inicial del sueño, en comparación con las siguientes, se describe como la más sensible y la que acumula las mayores alteraciones por estrés térmico (Okamoto et al. 2005). Estos autores demuestran la importancia de la temperatura en las primeras horas de la noche para el descanso durante las fases posteriores.

Pero también existe otro problema. Utilizando las series de datos semihorarios de temperatura de mayo a octubre entre 2006 y 2013, se han detectado diversos días en los que la temperatura mínima se ha producido antes de la media noche, en la franja comprendida entre las 23 y las $24 \mathrm{~h}$, aumentando la temperatura en las horas posteriores. En Ferrol este hecho tiene lugar durante un 15\% de las noches, en Porto un $8 \%$ y en Faro, al sur del Portugal, un $5 \%$. En todos estos casos la temperatura mínima del día no sería un indicador adecuado de noche tropical ni de estrés térmico nocturno.

Estas limitaciones en el uso de la temperatura mínima como indicador de noches con riesgo de estrés térmico nos han llevado a desarrollar y proponer dos nuevos indicadores de cara a mejorar la evaluación de este tipo de riesgo térmico. También nos ha motivado el hecho de que, paralelo al aumento de las olas de calor, en Europa los estudios pronostican un incremento de este tipo de noches cálidas y tropicales, como los que ya se han constatado en las últimas décadas (WMO 2009, EEA Report 2012, Olcina 2012, Donat et al. 2013).

\section{FUENTES DE INFORMACIÓN Y METODOLOGÍA}

Para utilizar los indicadores propuestos es necesario disponer de datos subhorarios de la temperatura del aire. Con ellos, el primer indicador se calcula sumando el número de horas durante la noche en las que se supera el umbral de $20^{\circ} \mathrm{C}$. Posteriormente se relativiza el valor obtenido en función del número total de horas nocturnas para poder comparar las distintas noches del año, obteniendo así un porcentaje. De esta forma podemos obtener el número de noches en las que el porcentaje de horas que han superado dicho umbral es igual o superior al $40 \%$, a las que denominaremos «noches cálidas», para diferenciarlas de las noches tropicales en las que el umbral de los $20^{\circ}$ se alcanza el $100 \%$ de las horas 
nocturnas. Teniendo en cuenta, como ya hemos explicado, que las primeras fases del sueño son las más sensibles y las que afectan en mayor medida el descanso del organismo, hemos considerado que aquellas noches en las que durante las primeras horas se superen $\operatorname{los} 20^{\circ} \mathrm{C}$, pueden generar estrés térmico y problemas en el sueño e , incluso, en la salud. Ésto puede ocurrir particularmente en la vertiente atlántica peninsular, donde las noches son normalmente frescas e, incluso, frías durante el periodo comprendido entre mayo y octubre, y temperaturas nocturnas superiores a $20^{\circ} \mathrm{C}$ constituyen un hecho poco habitual. De ahí que denominemos a éstas como noches cálidas.

El segundo es un índice que permitiría evaluar la intensidad del estrés térmico nocturno. Se obtiene a través de la suma de los valores diezminutales durante el periodo con temperaturas iguales o superiores a $20^{\circ} \mathrm{C}$, y dividiendo después por la duración total de la noche. En ambos índices la noche se define como el periodo comprendido entre la puesta y la salida del sol. Todo el proceso necesario para el cálculo de ambos indicadores se ha realizado con el sistema estadístico R-GNU (3.01). Para el cálculo de la variación del número de horas entre la puesta y la salida del sol, se ha usado el paquete Sun-methods \{maptools\}, que a su vez usa el algoritmo de la National Oceanic \& Atmospheric Administration (NOAA).

El área de estudio donde hemos puesto en práctica estos indicadores corresponde a la fachada atlántica ibérica, desde Ferrol, al norte de Galicia, hasta Faro, al sur de Portugal, pudiendo analizar así la relación existente entre la ocurrencia de noches cálidas y la latitud, teniendo siempre al océano Atlántico como factor geográfico común. Sin embargo, el estudio se centrará más en Galicia debido a la mayor disponibilidad de datos (Figura 1).

Figura 1

LOCALIZACIÓN DE LOS OBSERVATORIOS METEOROLÓGICOS UTILIZADOS

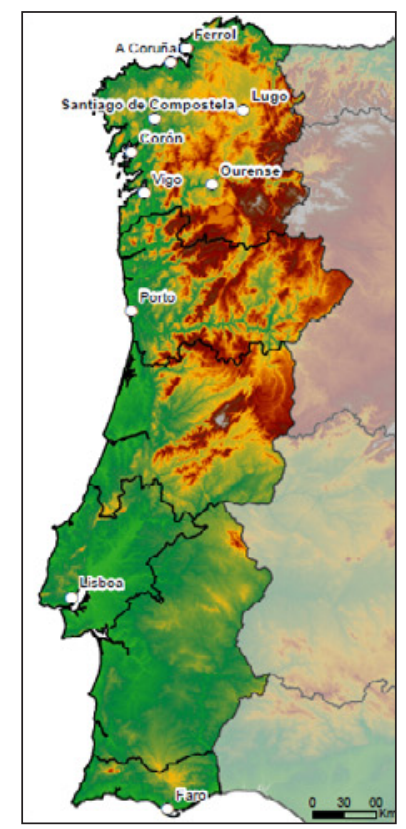


En el caso de Galicia se han utilizado los datos diezminutales del servicio meteorológico gallego, Meteogalicia, correspondientes a los meses de mayo a octubre. El periodo de estudio comprende 8 años, desde 2006 hasta 2013. La limitación al año 2006 se debe a la falta de datos diezminutales anteriores a dicha fecha para algunas estaciones. Se han seleccionado las siguientes estaciones meteorológicas: Ferrol, Lugo, Corón (Ría de Arousa), Santiago y Ourense. Asimismo, se han manejado los datos diarios de temperatura mínima para el periodo 1981-2013, recogidos por la la Agencia Estatal de Meteorología (AEMET) pero obtenidos gracias a la base de datos ECA (Home European Climate Assessment \& Dataset), con el objeto de conocer la evolución temporal de las noches tropicales en este intervalo de 33 años. Para ello se han utilizado los datos de las estaciones de Ourense, Vigo y A Coruña.

Para Portugal únicamente se ha podido disponer de datos semihorarios, correspondientes a Porto, Lisboa y Faro para el periodo 2006-2013, y han sido obtenidos del Integrated Surface Hourly (ISH) Dataset, disponible en el National Climatic Data Center (NOAA).

El análisis de las situaciones de isla de calor en Santiago y Ourense fueron realizadas con un termohigrómetro digital tipo Hanna y siguiendo la metodología seguida en los numerosos estudios de clima urbano en España (López et al. 1993, Moreno 1999).

\section{RESULTADOS}

\section{III.1. Frecuencias de noches cálidas y tropicales}

En la Tabla 1 podemos comprobar la importante diferencia que existe entre el número de noches tropicales y el de noches cálidas, tal y como las hemos definido en el apartado anterior. Lo primero que destaca es el bajo promedio de noches tropicales en las estaciones de Galicia, con valores que oscilan entre 0 en Lugo y 1,2 en Corón. Sin embargo, hay que resaltar el hecho de que estas noches tropicales constituyen un fenómeno caracterizado por una gran variabilidad temporal, como se puede observar en la Figura 2. En ella se representa la evolución del número de noches tropicales en tres ciudades gallegas entre 1981 y 2013 . En todas ellas se observa una fuerte irregularidad interanual, pudiendo registrarse en determinados años incluso hasta 10 y 12 noches tropicales; es el caso de Vigo y Ourense y de veranos tan cálidos como el de 1981 y, sobre todo, el de 2003, cuando se produjo la intensa ola de calor en agosto que provocó miles de muertos en toda Europa. Estos años con veranos muy calurosos se alternan con años en los que no se ha registrado ninguna noche tropical y, otros, más frecuentes, en los que tienen lugar entre 1 y 2 noches.

Como ocurre con muchos fenómenos climáticos extremos, la elevada variabilidad interanual es una de sus características más importantes, lo cual resta valor a los valores medios que ocultan una realidad más compleja y, en estos casos, unos episodios de riesgo que, de forma irregular, pueden afectar gravemente al bienestar y a la salud de la población.

Los bajos promedios de noches tropicales en Galicia se deben también al comportamiento de las temperaturas durante los días calurosos; éstas sufren importantes oscilaciones, con temperaturas máximas muy elevadas, como sería el caso de muchas zonas del interior (comarcas de Ourense o Santiago, por ejemplo), pero con un descenso térmico rápido a partir de la puesta de sol. Ello tiene como consecuencia inmediata el aumento de las sensaciones térmicas de frescor por parte de la población tras la retirada del sol. Este hecho se debe a la 
Tabla1

VALORES MEDIOS DE NOCHES TROPICALES Y NOCHES CÁLIDAS ENTRE MAYO Y OCTUBRE (2006-2013)

\begin{tabular}{|l|c|c|c|}
\hline & Noches tropicales & Noches cálidas & $\begin{array}{c}\text { Temperatura media de las } \\
\text { mínimas (may-oct) }\end{array}$ \\
\hline Ferrol & 1 & 5 & 12,7 \\
\hline Santiago & 0,3 & 3,2 & 10,8 \\
\hline Corón & 1,2 & 6,2 & 13,3 \\
\hline Ourense & 1,1 & 13,2 & 11,9 \\
\hline Lugo & 0 & 0,6 & 9,5 \\
\hline Porto & 6,2 & 14,5 & 13,9 \\
\hline Lisboa & 34 & 73 & 15,7 \\
\hline Faro & 72 & 113 & 16,3 \\
\hline
\end{tabular}

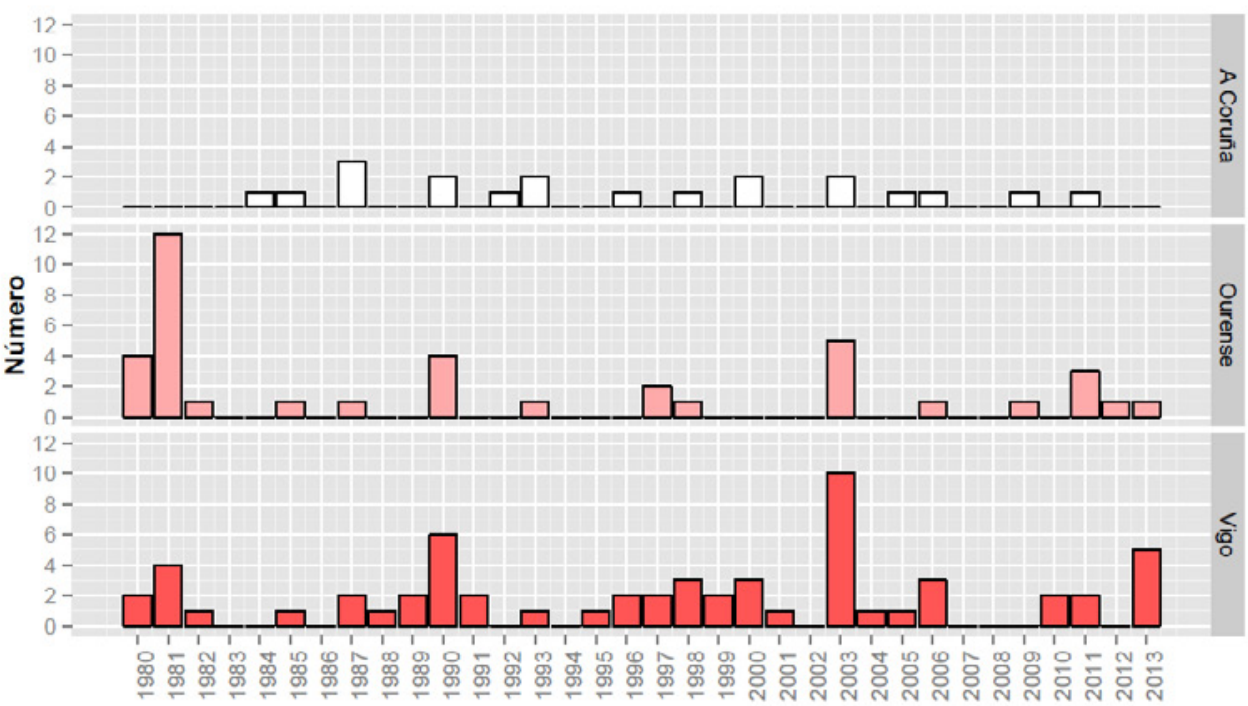

densa cubierta vegetal que cubre el territorio gallego; durante el día, a pesar del bajo albedo, acumula muy poca energía, pues buena parte se gasta en los procesos de evapotranspiración; es por ello que, al caer el sol, la escasa energía acumulada en forma de calor se disipa enseguida y las temperaturas desciendan de forma rápida. Este comportamiento térmico es, sin embargo, muy diferente en el centro de las ciudades. El uso masivo de granito para la construcción así como de otros materiales urbanos hace que se acumule gran cantidad de calor durante el día; éste mantendrá unas temperaturas más altas durante buena parte de la noche, generando el fenómeno de isla de calor que va a incrementar el número de noches cálidas y tropicales en el interior de las ciudades, como veremos más adelante. 
Este comportamiento térmico durante los días calurosos es el que explica que lugares como Ourense, con temperaturas máximas muy elevadas en numerosas ocasiones durante el verano (Royé y Martí, 2012), tenga un número tan escaso de noches tropicales en las que la temperatura mínima sea superior a $\operatorname{los} 20^{\circ} \mathrm{C}$. Sin embargo el número medio de noches cálidas es de 13,2, lo que refleja mucho mejor la realidad térmica estival de la depresión de Ourense, en la que el estrés por calor es bastante habitual durante, al menos, la primera mitad de la noche. En menor medida este comportamiento se produce en otras áreas del interior de Galicia, como es el caso del entorno de Santiago. Frente a 0,3 noches tropicales se producen 3,2 noches cálidas. En el caso de Lugo, su situación geográfica favorece unas temperaturas máximas y mínimas más frescas durante los meses y días más cálidos; no es de extrañar pues que no se registre ninguna noche tropical, y tan solo una media de 0,6 noches cálidas por año.

Figura 3

FRECUENCIA DE NOCHES CÁLIDAS ENTRE 2006 Y 2013

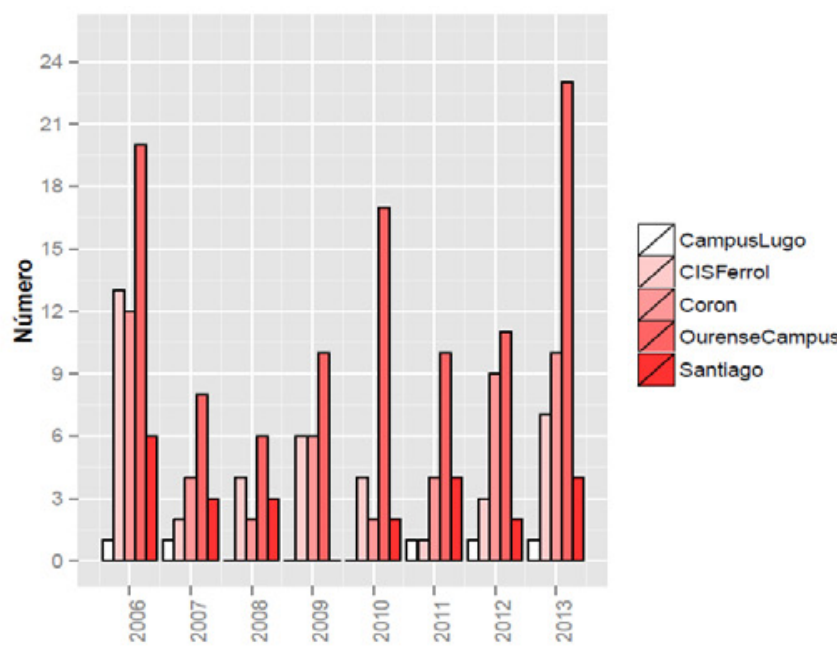

Diferente es el comportamiento de los espacios litorales, donde las oscilaciones térmicas diarias durante los meses de verano son mucho más suaves como resultado del efecto regulador del océano. Es por ello que, aunque la frecuencia media de noches tropicales es también muy bajo, no haya tanta diferencia con el número de noches cálidas como ocurre en los territorios interiores. Sin embargo, el efecto de la latitud y, por ende, de la mayor influencia de las masas de aire cálidas subtropicales, sí que van a ser determinantes en el incremento del riesgo por estrés térmico asociado a las noches tropicales y cálidas. Conforme descendemos por el litoral atlántico, el número de noches calurosas aumenta de forma constante y rápida (Tabla 1). Así, si en la ría de Arousa se registra una media de 1,2 noches tropicales y 6,2 noches cálidas, en Porto la frecuencia es ya de 6 y 14,5 noches respectivamente. En Lisboa se produce un fuerte ascenso, con 34 noches tropicales y 73,3 noches cálidas, que continúa hasta Faro donde se registran 72 y 113 noches respectivamente. 
Analizando la serie temporal comprendida entre 1980 y 2013, no se observa ninguna tendencia significativa de aumento del número de noches tropicales (Figura 2). Tampoco se aprecia en la evolución de las noches cálidas en los últimos 8 años del estudio (Figura 3), si bien son muy pocos años como para poder hablar de tendencias. Lo que si se observa claramente es una elevada variabilidad interanual, que caracteriza también a las noches tropicales. Destacan los datos de Ourense, que oscila entre las 6 noches cálidas de 2008 y las 23 noches que se registraron durante los meses centrales de 2013. También Corón, en la Ría de Arousa, o Ferrol, en el Golfo Artabro, presentan una elevada irregularidad, con valores que oscilan entre 2 y 12 en el caso de Corón, y de 1 a 13 en el caso de Ferrol. Santiago oscila entre 0 y 6 , y Lugo sólo ha registrado en 5 ocasiones una noche cálida.

En cuanto al periodo con mayor riesgo de noches calurosas, éste se concentra entre junio y septiembre, si bien son los meses de julio y agosto los que padecen un mayor número de ellas (Figura 4). En las dos estaciones litorales, Ferrol y Corón, la media es de dos noches cálidas por mes, mientras que en Ourense éstas ascienden hasta 5 en los dos meses centrales del verano. Es en estos meses cuando tienen lugar con una mayor frecuencia situaciones atmosféricas de estabilidad así como la advección de masas de aire cálidas procedentes del interior de la Península o del norte de África que suelen generar episodios de calor (Lorenzo et al. 2008, Martí et al. 2011).

Figura 4

FRECUENCIA MENSUAL DE LAS NOCHES CÁLIDAS

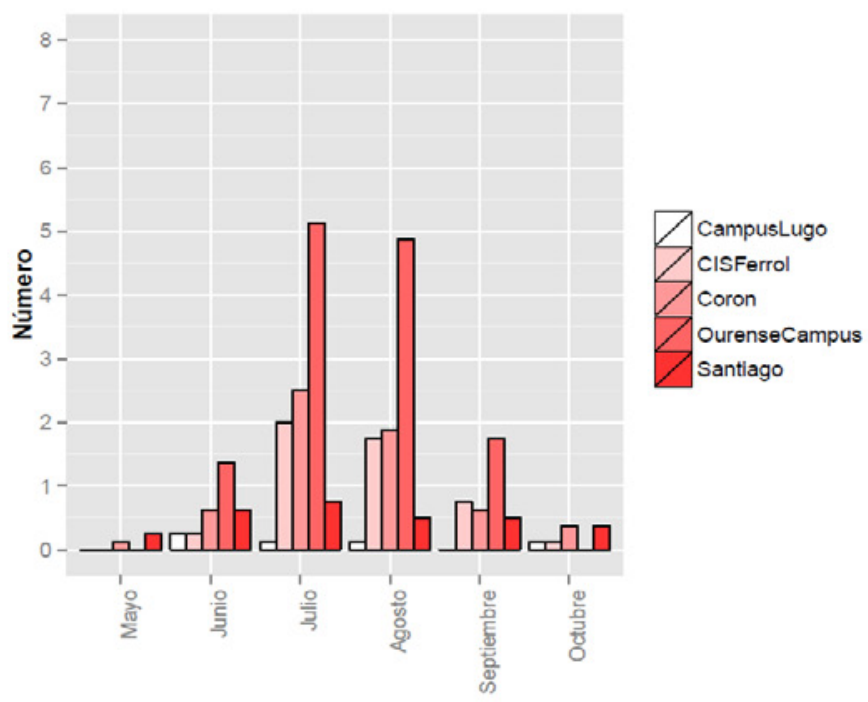

\section{III.2. La persistencia del calor nocturno}

Si, como vimos anteriormente, el número de noches cálidas aumenta hacia el sur, la persistencia de los elevados valores térmicos nocturnos también tiene una relación directa con la latitud. En la Figura 5 está representada la distribución de las noches cálidas en funcion 
del porcentaje de horas nocturnas en las que se superaron los $20^{\circ} \mathrm{C}$. Vemos como de norte a sur la dispersión experimenta un continuo descenso. En el caso de las estaciones portuguesas la mitad de las noches se concentran en la parte superior, con medianas que superan el $90 \%$ o alcanzan el $100 \%$. En Lisboa, por ejemplo, la mitad de las noches cálidas se caracterizan por registrar durante más del $95 \%$ de las horas temperaturas por encima de los $20^{\circ} \mathrm{C}$; y en Faro más de la mitad de estas noches son tropicales, con el 100\% de su duración por encima del umbral de $20^{\circ} \mathrm{C}$. Las medianas van descendiendo hacia el norte, y así Porto tiene una mediana del $90 \%$ y Corón, ya en Galicia, del 62\%. Sin embargo Ferrol, la estación litoral más septentrional, tiene un comportamiento más similar a Porto en cuanto a la distribución de las noches, con una mediana del 89\%. Es decir, casi la mitad de las noches cálidas que tienen lugar aquí, aunque pocas en número, son tropicales. La explicación podríamos encontrarla en el hecho de que el noroeste de Galicia se ve afectado con menor frecuencia por masas de aire muy cálidas, pero cuando éstas alcanzan latitudes tan septentrionales lo hacen asociadas a episodios de calor más intenso que favorecen noches anómalamente calurosas, muchas de ellas tropicales. Mientras que en regiones más meridionales, con temperaturas mínimas medias bastante más altas (Tabla 1), no hace falta que se produzcan este tipo de episodios de calor más extremos para que tengan lugar noches cálidas y tropicales.

Figura 5

DISTRIBUCIÓN DEL TOTAL DE NOCHES CÁLIDAS ENTRE 2006 Y 2013 EN FUNCIÓN

DE LA PERSISTENCIA DE TEMPERATURAS $>20^{\circ}$

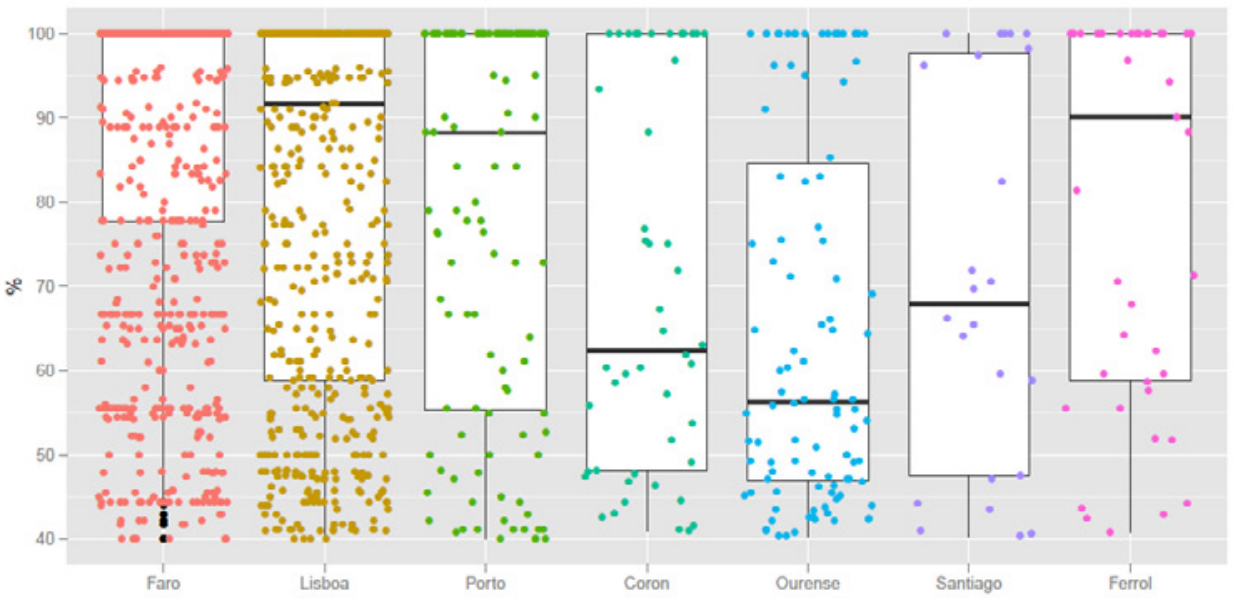

En zonas de interior, como Ourense o Santiago, el comportamiento es diferente. La dispersión es moderada, pero con los dos tercios de las noches cálidas concentrados en porcentajes inferiores al 80\%; ello significa que la persistencia de altas temperaturas por la noche es menor, por la mayor rapidez con la que descienden las temperaturas nocturnas en estas zonas más continentales, como ya se explicó. En el caso de Ourense, con un elevado número de noches cálidas, la mitad de ellas registran temperaturas superiores a $20^{\circ} \mathrm{C}$ sólo durante la primera mitad de la noche, con un mayor enfriamiento a lo largo de la otra mitad. 


\section{III.3. La intensidad de las noches cálidas y el efecto de la isla de calor urbana}

Para evaluar la intensidad del calor durante las noches cálidas se ha utilizado un índice obtenido a través de la suma de las temperaturas diezminutales a lo largo del periodo nocturno con valores iguales o superiores a $20^{\circ} \mathrm{C}$, y dividido después por la duración total de la noche. En las Figuras 6 y 7 se representa la distribución de las noches cálidas según tres niveles de intensidad. Las noches con una intensidad de calor baja serían aquellas con un índice inferior a 70, lo que equivaldría aproximadamente a una temperatura media nocturna inferior a $21^{\circ} \mathrm{C}$; las noches con una intensidad media corresponderían a aquellas que tienen un índice entre 70 y 130 , y que tendrían unos valores medios de temperatura entre $21^{\circ} \mathrm{C}$ y $23^{\circ} \mathrm{C}$; y finalmente, las de intensidad alta, con un índice superior a 130 y que se caracterizarían por tener unas temperaturas medias nocturnas superiores a $23^{\circ} \mathrm{C}$.

Figura 6

INTENSIDAD DE LAS NOCHES CÁLIDAS

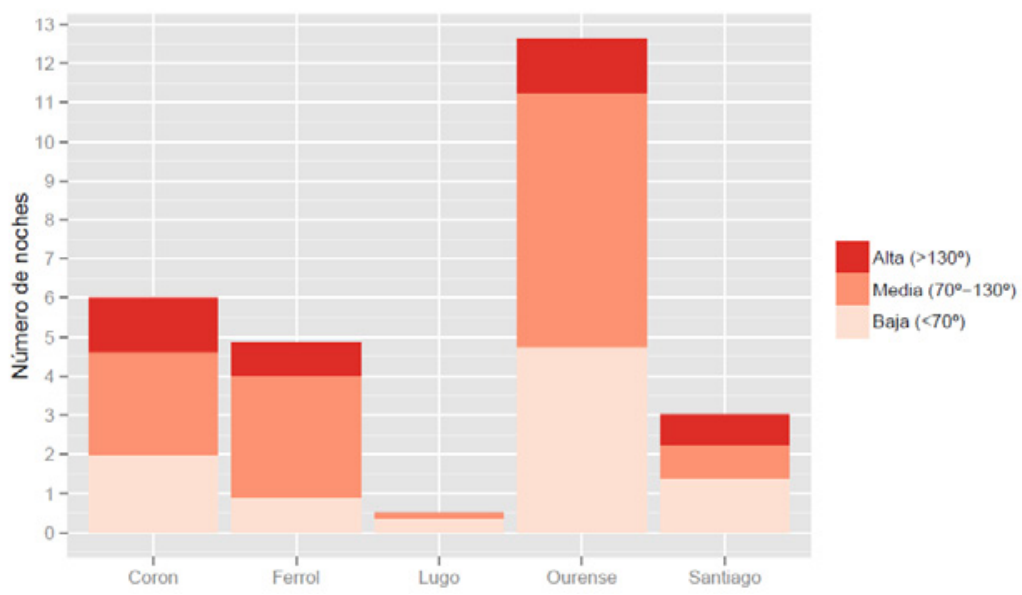

Observamos como las noches con mayor intensidad de calor son las menos frecuentes, pues el riesgo de estrés térmico es elevado, si tenemos en cuenta que las temperaturas nocturnas son muy altas $\left(24,5^{\circ} \mathrm{C}\right.$ es la temperatura media de todo este grupo de noches cálidas). Son más escasas, sólo en términos relativos, en las zonas de interior como Ourense (9\% del total de las noches cálidas) y Santiago (13\%). En las áreas litorales, sin embargo, las noches de calor intenso representan más del 20\% del total, llegando a ser la cuarta parte de las noches cálidas que tienen lugar en la Ría de Arousa. Mucho más frecuentes son las noches con intensidades medias, en las que la temperatura estaría comprendida aproximadamente entre $21^{\circ} \mathrm{C}$ y $23^{\circ} \mathrm{C}$. Finalmente, las de intensidad baja constituyen también un porcentaje importante, especialmente en las zonas de interior de Galicia (40\% en el caso de Ourense).

Pero estas intensidades, así como las frecuencias de noches cálidas, pueden aumentar moderadamente en el centro de las áreas urbanas. Las ciudades, como ya sabemos por la abundante bibliografía existente, constituyen focos de calor generados por diversos facto- 
res de carácter local (López Gomez et al. 1993, Fernández et al. 1998, Moreno 1999). Los materiales urbanos con una mayor capacidad calorifica, el complejo entramado de la ciudad y la disminución del Sky View Factor, la generación de calor antrópico con el tráfico o la iluminación, el menor consumo de calor sensible por evapotranspiración, o la contaminación del aire y el efecto invernadero a escala local, son los responsables de que las ciudades se comporten en muchos momentos del día y del año como islas de calor en comparación a su entorno próximo, especialmente durante la noche. Por ello, a la hora de evaluar el riesgo de estrés por calor nocturno para la población, es necesario tener en cuenta las importantes modificaciones que este fenómeno climático urbano puede tener sobre los indicadores que se utilicen, como en nuestro caso el de las noches cálidas. Además, hay que tener en cuenta que la mayor parte de las estaciones meteorológicas urbanas están ubicadas en la periferia de las ciudades, donde los factores locales citados son muy diferentes a los del centro. Vamos a ver a continuación dos ejemplos muy claros correspondientes a Santiago y Ourense.

Figura 7

NÚMERO DE NOCHES CÁLIDAS EN FUNCIÓN DEL ÍNDICE DE INTENSIDAD

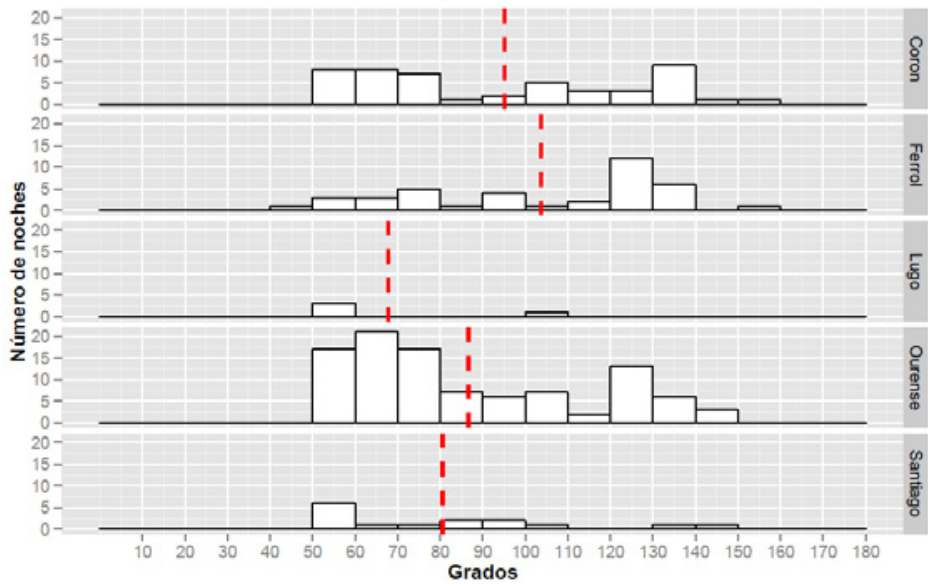

Entre el 4 y el 8 de julio de 2013 Galicia y buena parte del resto de España se vieron afectadas por una intensa ola de calor. En muchos lugares de la mitad meridional de Galicia se superaron durante varios días $\operatorname{los} 35^{\circ} \mathrm{C}$ e, incluso, los $40^{\circ} \mathrm{C}$. Durante esos días se batieron varios records térmicos, como las máximas de Ferreira de Pantón, al sur de Lugo, $\left(43,1^{\circ} \mathrm{C}\right)$ y la de Pontevedra $\left(37^{\circ} \mathrm{C}\right)$; o la temperatura mínima de Vigo del día $7\left(27,5^{\circ} \mathrm{C}\right)$. Los valores mínimos se mantuvieron en muchas zonas por encima de los $20^{\circ}$ durante varios días, especialmente en las áreas costeras. Fue un episodio de calor intenso que provocó una situación de estrés térmico durante varios días, tanto en las horas diurnas como en las nocturnas (Figura 8). Las noches tropicales se sucedieron durante 4 días seguidos en muchas localidades; éste fue el caso de Vigo y Corón, en las Rías Baixas (Figura 9). Incluso Ferrol, al noroeste, registró una noche tropical precedida por dos noches cálidas, casi tropicales, pues durante más del $90 \%$ de éstas las temperaruras no bajaron de los $20^{\circ} \mathrm{C}$. 
Figura 8

EVOLUCIÓN DE LAS TEMPERATURAS EN VIGO, SANTIAGO Y OURENSE DURANTE LA OLA DECALOR DE JULIO DE 2013

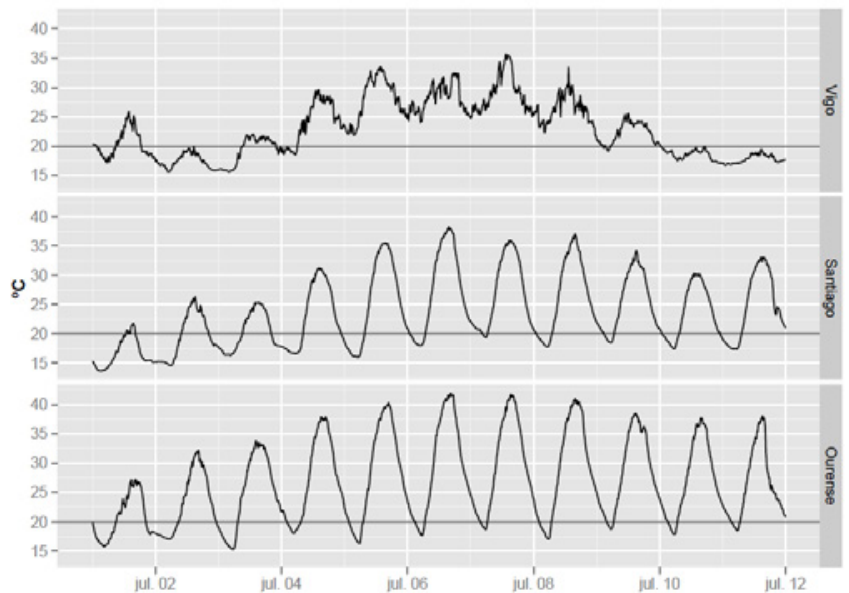

Figura 9

PERSISTENCIA DE LAS TEMPERATURAS NOCTURNAS $>20^{\circ}$ DURANTE LAS NOCHES DE LA OLA DE CALOR DE JULIO DE 2013. EN NEGRO NOCHES TROPICALES.
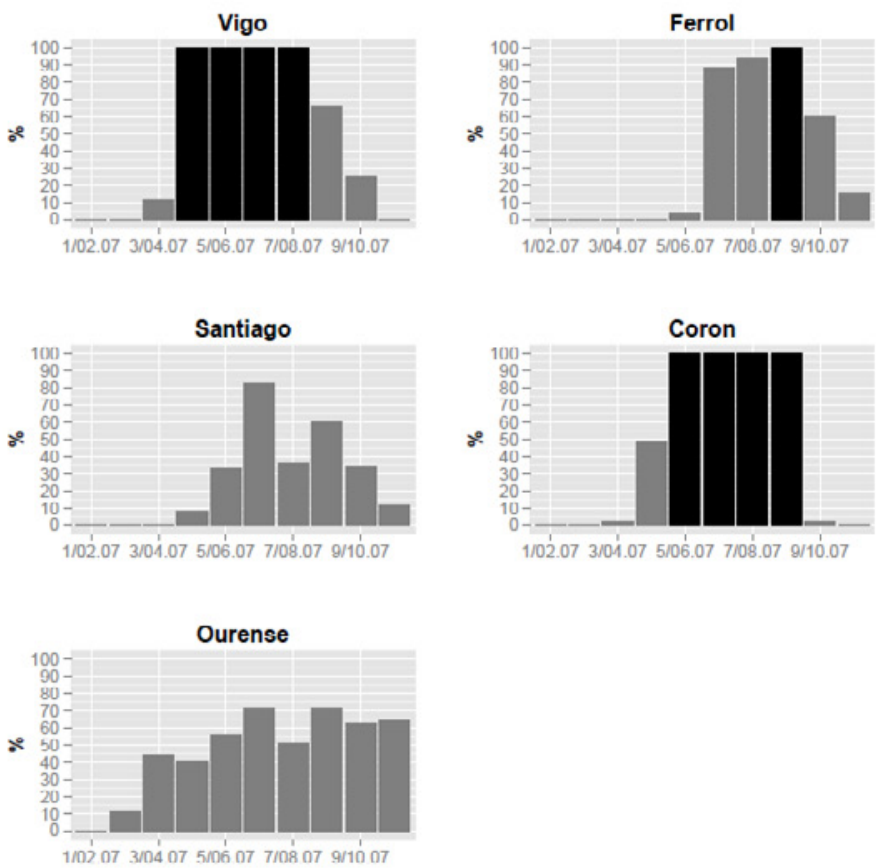
Ourense y Santiago, sin embargo, no registraron ninguna noche considerada como tropical, por lo que cabría pensar que la población de ambas ciudades no sufrió tanto estrés por calor, al menos si tenemos en cuenta sólo las temperaturas mínimas. Sin embargo, durante varios días seguidos (8 en el caso de Ourense) sus habitantes soportaron noches cálidas. Debido al más rápido enfriamiento de las temperaturas por su alejamiento del océano, las altas temperaturas de las primeras horas de la noche no persistieron hasta el amanecer, cuando las mínimas descendieron de $\operatorname{los} 20^{\circ} \mathrm{C}$.

Pero este comportamiento térmico corresponde al entorno de las estaciones meteorológicas, ubicadas las dos en los campus que se encuentran en los límites de las ciudades, con una baja densidad constructiva y un elevado porcentaje de superficie vegetal. Muy diferentes fueron las condiciones bioclimáticas en el centro de ambas ciudades. En la Figura 10 está representada la configuración de la isla de calor que había en Santiago a las 24 h del día 8 de julio de 2013, en plena ola de calor. Observamos cómo mientras la periferia de la ciudad registraba temperaturas por debajo de $\operatorname{los} 23^{\circ} \mathrm{C}$, el centro urbano se encontraba inmerso en un ambiente más caluroso, con temperaturas superiores a $\operatorname{los} 25^{\circ} \mathrm{C}$ y $26^{\circ} \mathrm{C}$. El uso masivo de granito para la construcción de las ciudades gallegas contribuye en buena medida a la generación y mantenimiento de estas islas de calor. En un estudio realizado en Santiago (Martí y Miragaya, 1998) se demuestra la importancia que la geometría urbana y el material constructivo predominante, el granito, tenían en la generación del ambiente térmico de la ciudad. A primeras horas de la tarde de un día soleado de julio las superficies de granito de algunas de las plazas de la ciudad alcanzaban los $56^{\circ} \mathrm{C}$, y a las 24 h todavía se mantenían con valores en torno a los $35^{\circ} / 40^{\circ} \mathrm{C}$. Esta energía acumulada durante el día mantendrá, tras la puesta del sol, unas temperaturas más altas toda la noche en el centro de la ciudad, lo que va a incrementar considerablemente el número de noches cálidas y tropicales que afectarán al bienestar y a la salud de la población.

Con esta situación a media noche, cuando la estación meteorológica del campus registraba $22,1^{\circ} \mathrm{C}$, la población del Casco historico y del Ensanche estaba soportando un ambiente de fuerte estrés por calor, con temperaturas $3^{\circ} \mathrm{C}$ y $4^{\circ} \mathrm{C}$ más elevadas. La temperatura de la estación fué descendiendo hasta alcanzar una mínima de $18,5^{\circ} \mathrm{C}$; sin embargo, algunos sectores del centro posiblemente se encontrarían al amanecer todavía con temperaturas superiores a los $22^{\circ} \mathrm{C}$, claro indicador de noche tropical.

Una situación similar ocurrió en Ourense el 17 de julio de 2006 (Figura 11). Tras una noche muy calurosa $\left(26,6^{\circ} \mathrm{C}\right.$ a las $00 \mathrm{~h}$ del día 17$)$, la estación meteorológica situada en el Campus As Lagoas de Ourense registró una mínima de 20,8 a las 06 h. Sin embargo, el centro de la ciudad permanecía al amanecer todavía con temperaturas por encima de $\operatorname{los} 23^{\circ} \mathrm{C} \mathrm{y}$ $24^{\circ} \mathrm{C}$, lo que supuso una agobiante situación de estrés por calor que afectó a una buena parte de los habitantes de la ciudad. Ese mismo día la maxima alcanzó los $41,5^{\circ} \mathrm{C}$. Si tenemos en cuenta que éste día fue el último de un episodio de calor intenso que desde el día 13 mantuvo las máximas por encima de $\operatorname{los} 35,5^{\circ} \mathrm{C}$ y las mínimas por encima de $20^{\circ} \mathrm{C}$, podemos hacernos una idea del fuerte estrés térmico que sufrió la población de Ourense esos días, en especial la que vive en el centro urbano.

La configuración y la intensidad de las islas de calor que se forman en el interior de las ciudades provocan que no siempre coincidan los valores térmicos recogidos en las estaciones meteorológicas, ubicadas habitualmente en la periferia urbana, con las verdaderas temperaturas que los habitantes de los centros urbanos están percibiendo, como se ha demostrado en San- 
Figura 10

ISLA DE CALOR DE SANTIAGO DE COMPOSTELAA LAS 24 H DEL DÍA 8 DE JULIO DE 2013.

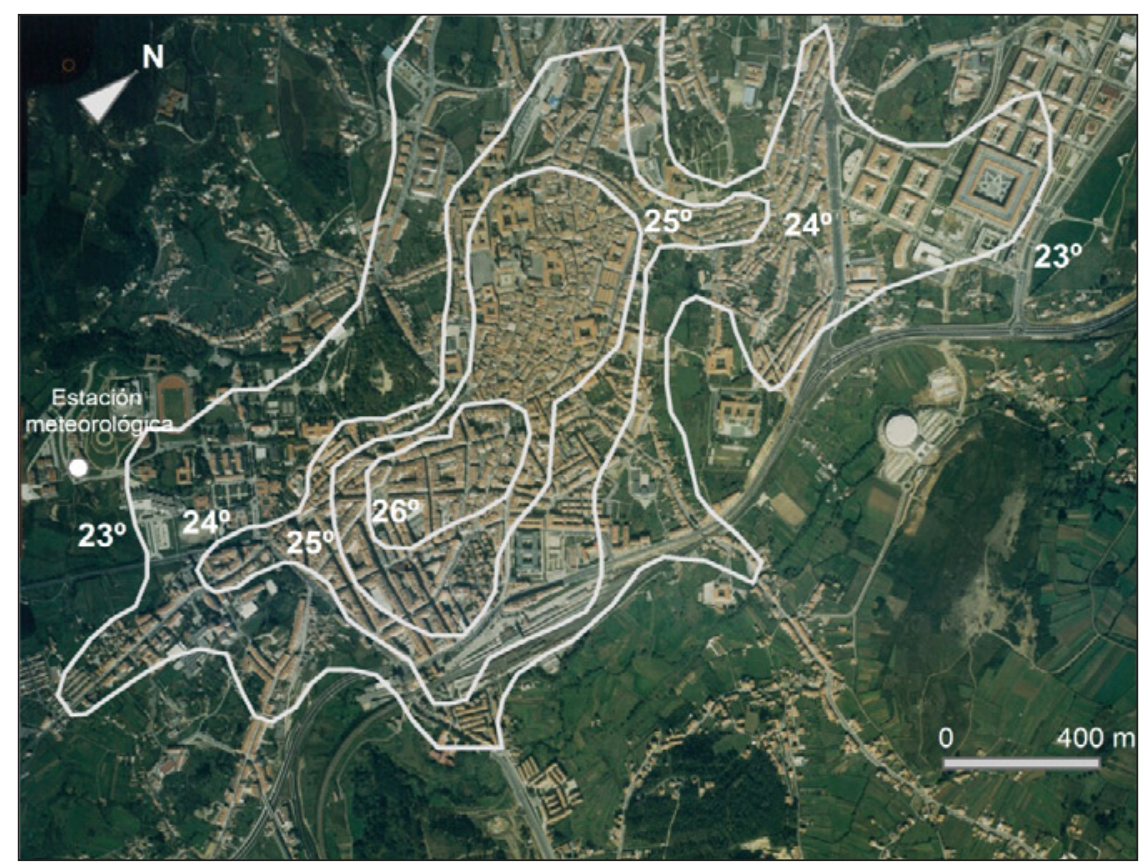

Figura 11

ISLA DE CALOR DE OURENSE A LAS 06 H DEL DÍA 17DE JULIO DE 2006.

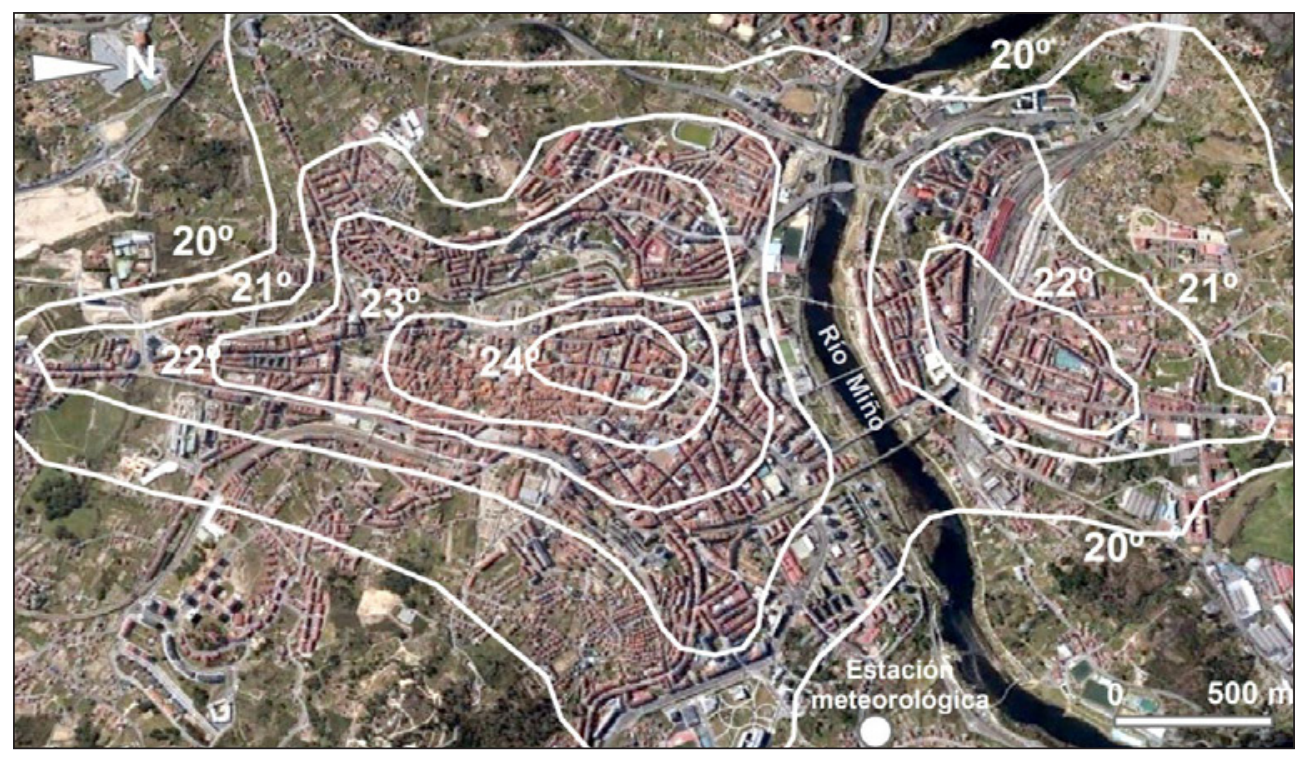


tiago y Ourense. Las noches cálidas son, por ello, más frecuentes e intensas en el centro de las ciudades, por lo que sus habitantes sufren mayores sensaciones térmicas de estrés que pueden afectar a su bienestar y a su salud. Es necesario profundizar en trabajos posteriores en el análisis de este hecho, para poder cuantificar de una forma más precisa los efectos de las islas de calor sobre la frecuencia, la intensidad y la persistencia de las noches cálidas en las áreas urbanas.

\section{CONCLUSIONES}

Con la aplicación del método de estudio propuesto se ha comprobado que un importante número de noches con posible estrés térmico en la primera mitad de la noche queda oculto si solamente se tienen en cuenta las temperaturas mínimas como indicador. La utilización de datos subhorarios permite valorar con más detalle las características térmicas de las noches entre mayo y octubre, pudiendo así evaluar con más precisión el riesgo para el bienestar y la salud de la población. Hay que tener en cuenta, además, que son las primeras horas de la noche y la primera fase del sueño la que se describe como la más sensible y la que acumula las mayores alteraciones por estrés térmico.

Los resultados del análisis han constatado un aumento continuado de la frecuencia de noches tropicales y de noches cálidas en la fachada atlántica ibérica, desde el norte de Galicia hasta el sur de Portugal. La menor latitud y la proximidad al litoral están directamente relacionados con la mayor persistencia del calor y del estrés térmico durante las noches cálidas. En zonas más continentales, a veces con mayor frecuencia de noches cálidas, la persistencia del calor es, sin embargo, menor, debido al más rápido enfriamiento de las temperaturas.

El noroeste de Galicia se ve afectado con menor frecuencia por masas de aire muy cálidas, pero cuando éstas alcanzan latitudes tan septentrionales lo hacen normalmente asociadas a episodios de calor más intensos que favorecen noches anómalamente calurosas, muchas de ellas tropicales. Mientras que en regiones más meridionales, con temperaturas mínimas medias bastante más altas, no hace falta que se produzcan este tipo de episodios de calor más extremos para que tengan lugar noches cálidas y tropicales.

\section{BIBLIOGRAFÍA}

ALEXANDER L.V. Y ZHANG X. (2006): «Global observed changes in daily climate extremes of temperature and precipitation», Journal of Geophysical Research, 111, D05109.

BUGUET A. (2007): «Sleep under extreme environments: Effects of heat and cold exposure, altitude, hyperbaric pressure and microgravity in space», Journal of the Neurological Sciences, 262:145-152.

COUMOU, D. Y RAHMSTORF, S. (2012): «A decade of weather extremes», Nature Climate Change, 2:491-496.

COUMOU, D.Y ROBINSON, A. (2013): «Historic and future increase in the global land area affected by monthly heat extremes», Environ. Res. Lett., 8 :034018.

DÍAZ, J. (2006): «Impact of extreme temperatures in daily mortality in Madrid between 45 and 64». International Journal of Biometeorology, 50, pp. 342-356.

DÍAZ, J. (2002): «Heat waves in Madrid, 1986-1997: effects on the health of the elderly». Int. Arch. Occup. Envirnonment Health, 75, pp. 163-175. 
DONAT, M.G. (2013): «Updated analyses of temperature and precipitation extreme indices since the beginin of the twentieth century». Journal of Geophysical Research: Atmospheres. Vol 118, pp. 1-16.

DWD (2013): Deutsche Wetterdienst - Wetterlexikon (Servicio Alemán de Meteorología - enciclopediameteorológica), http://www.dwd.de/bvbw/appmanager/bvbw/dwdwwwDesktop?_nfpb=true\&_pageLabel=dwdwww_menu2_wetterlexikon\&_nfls=false, 25/09/2013.

EEA Report (2012): Urban adaptation to climate change in Europe: Challenges and opportunities for cities together with supportive national and European policies, $\mathrm{Nr} 2$, Copenhagen: European Environment Agency.

FERNÁNDEZ, F., GALÁN, E. y CAÑADA, R.(Coords.) (1998): Clima y ambiente urbano en ciudades ibéricas e iberoamericanas. Ed. Parteluz, Madrid. 606 pp.

FISCHER, E.M. Y SCHÄR, C. (2010): «Consistent geographical patterns of changes in high-impact European heatwaves», Nature Geoscience, 3:398-403.

FOUILLET ,A., REY, G., LAURENT, F., PAVILLON, G., BELLEC, S., GUIHENNEUCJOUYAUX, C., CLAVEL, J., JOUGLA, E. Y HÉMON, D. (2006): «Excess mortality related to the August 2003 heat wave in France», Int Arch Occup Environ Health, 80:16-24.

GARCÍA HERRERA, R. (2005): «Extrem summer temperaturas in Iberia: health impacts ans associated synoptic conditions». Annals of Geophysics, 23, pp. 239-250.

GRIZEA, L., HUSSA, A., THOMMENA O., SCHINDLERA, C.Y BRAUN-FAHRLÄNDER, C. (2005): «Heat wave 2003 and mortality in Switzerland», Swiss Med WKLY, 135:200-205.

HASKELLA, E.H., PALCAA, J.W., WALKERA, J.M., BERGERA, R.J. y HELLERA, H.C. (1981): «The effects of high and low ambient temperatures on human sleep stages», Electroencephalography and Clinical Neurophysiology, 51:494-501.

HONG KONG OBSERVATORY (2012): http://www.hko.gov.hk/cis/regione.htm, 25/09/2013.

HÖPPE, P. Y MARTINAC, I. (1998): «Indoor climate and air quality. Review of current and future topics in the field of ISB study group 10», Int J Biometeorol, 42:1-7.

HUYNENM, M. y MARTENS, P. (2001): «The impact of heat waves and cold spells on mortality rates in the Dutch population». Environ Health Perspect, 109, pp. 463-470.

IPCC (2014): Impacts, Adaptation and Vulnerability. Working Group II Contribution to AR5.

KOPPE, C., KOVATS, S., JENDRITZKY, G. Y MENNE, B. (Ed.) (2004): Heat-waves: risks and responses, WHO Regional Office for Europe, Page 30.

LISA, V. (2009): «Climate extremes: progress and future directions». International Journal of Climatology. Vol. 29, issue 3, pp. 317-319.

LÓPEZ, A., FERNÁNDEZ, F., ARROY, F., MARTIN VIDE, J. y CUADRAT, J.M. (1993): El clima de las ciudades españolas. Cátedra. Madrid, 268 pp.

LORENZO, M.N., TABOADA, J. y GIMENO, L. (2008): «Links between circulation weather types and teleconnection patterns and their incluence on precipitation patterns in Galicia (NW Spain)». International Journal of Climatology, 28, pp. 1493-1505.

MARTÍ, A., CABALAR, M. y GARCÍA, E. (2011): «Natureza e medio ambiente». En PIÑEIRA, M.J. Y SANTOS, X.M. (Coords.): Xeografía de Galicia. Xerais. Vigo, pp. 99-129.

MARTI, A. y MIRAGAYA, A. (1998): «Geometría urbana, temperaturas e isla de calor en Santiago de Compostela». En FERNÁNDEZ et al. Clima y ambiente urbano en ciudades ibéricas e iberoamericanas. Ed. Parteluz, Madrid. pp. 207-218. 
MATZARAKIS, A. y MAYER, H. (1996): «Another kind of environmental stress: thermal stress», WHO Newsletter, 18:7-10.

MATZARAKIS, A., RUTZ, F. y MAYER, H. (2006): «Modelling the thermal bioclimate in urban areas with the RayMan Model», En: Conference Proceedings of the $23^{\text {rd }}$ Conference on Passive and Low Energy Architecture, Genova.

MATZARAKIS, A., RUTZ, F. y MAYER, H. (2007): «Modelling radiation fluxes in simple and complex environments - application of the RayMan model», International Journal of Biometeorology, 51:323-334.

MORENO GARCÍA, M.C. (1999): Climatología urbana. Edicions Universitat de Barcelona. $71 \mathrm{pp}$.

MUZET, A. (2007): «Environmental noise, sleep and health», Sleep Medicine Reviews, $11: 135: 142$.

NASTOS, P.ÇT. y MATZARAKIS, A. (2008): Human-Biometeorological effects on sleep disturbances in Athens, Greece: A Preliminary Evaluation», Indoor Built Environment, 17:535-542.

O’NEILL, M.S., ZANOBETTI, A. y SCHWARTZ, J. (2005): «Disparities by Race in HeatRelated Mortality in Four US Cities: The Role of Air Conditioning Prevalence», Journal of Urban Health, 82:191-197.

OKAMOTO-MIZUNO, K. y MIZUNO, K. (2012): «Effects of thermal environment on sleep and circadian rhythm», Journal of Physiological Anthropology, 31:1:14.

OKAMOTO-MIZUNO, K., TSUZUKI, K. y MIZUNO, K. (2005): «Effects of humid heat exposure in later sleep segments on sleep stages and body temperature in humans», Int $J$ Biometeorol, 49:232-237.

OLCINA, J. y MARTIN, D. (2012): «Variaciones en la densidad del oxígeno en el aire y su influencia sobre la salud humana». Boletín de la Asociación de Geógrafos Españoles, ${ }^{\circ}$ 58, pp. 7-32.

ROONEY, C., MCMICHAEL, J.A., KOVATS, R.S. y COLEMAN, M.P. (1995): «Excess mortality in England and Wales, and in Greater London, during the 1995 heatwave», $J$ Epidemiol Community Health, 52:482-486.

ROYÉ, D., MARTI, A. y CABALAR, M. (2012): «Aproximación al comportamiento espacial del estrés térmico en Galicia a través de luso del índice bioclimático PET». RODRÍGUEZ PUEBLA et al. (Eds.) : Cambio climático. Extremos e impactos. Publicaciones de la Asociación Española de Climatología.Serie A, nº 8. Salamanca. pp 941-949.

RUSSO, S. y STERL, A. (2011): «Global changes in indices describing moderate temperature extremes from the daily output of a climate model». Journal of Geophysical Research: Atmospheres. Vol. 116, issue db, 16.

VINCENT L.A. y PETERSON T.C. (2005): «Observed Trends in Indices of Daily Temperature Extremes in South America 1960-2000», AMS Journal of Climate, 18:5011-5023.

WHO (2004): Heat waves: risk and reponses. Series 2, World Health Organization. Copenhagen. $120 \mathrm{pp}$.

WMO (2009): Analysis of extremes in a changing climate in support of informed decisions for adaptation. World Meteorological Organization. Geneva. WCDMP-No. 72, 52 pp. 